\title{
A Practice-led Approach to Facial Animation Research
}

\author{
Robin J.S. Sloan ${ }^{1}$, Brian Robinson ${ }^{1}$, Ken Scott-Brown ${ }^{2}$, Fhionna Moore ${ }^{2}$, and Malcolm Cook ${ }^{2}$ \\ Institute of Arts, Media and Computer Games ${ }^{1}$, School of Social and Health Sciences ${ }^{2}$ \\ University of Abertay Dundee \\ r.sloan@abertay.ac.uk
}

\section{Background and Methodology}

In facial expression research, it is well established that certain emotional expressions are universally recognized. Studies into the observer perception of dynamic expressions have built upon this research by highlighting the importance of particular facial regions, timings, and temporal configurations. In many studies, the stimuli for such studies have been generated through posing by non-experts or performances by trained actors. However, skilled character animators are capable of crafting recognizable, believable emotional facial expressions as a part of their professional practice. 'Emotional Avatars' was conceived as an interdisciplinary research project which would draw upon the knowledge of animation practice and emotional psychology. The aim of the project was to jointly investigate the artistic generation and observer perception of emotional expression animation to determine whether the nuances of emotional facial expression could be artistically choreographed to enhance audience interpretation.

The core idea was that traditional animation principles (Thomas and Johnston, 1981) ought to play a more crucial role in animation research. Rather than depending solely upon the established quantitative and qualitative methods, the Emotional Avatars research was practice-led, and much of the initial research was carried out in the studio rather than the laboratory. Sullivan's (2009) discursive method - which incorporates visual arts practice, empirical methods, and interpretive methods - served as the framework for the Emotional Avatars methodology. A triparadigm methodology was then devised specifically for the project. The methodology placed animation practice at the forefront of the research, as dictated by Sullivan's framework. Practice-based animation studies were conducted in the studio in order to experiment with facial animation production and to form subjective predictions. The outputs of these studies (including the predictions) were put to the test in subsequent quantitative and qualitative studies. The quantitative studies sought to assess observer perception of facial animation and test predictions, while the qualitative studies involved groups of animators in detailed explorations of animation practice and observation.

\section{Results and Future Work}

After early periods of practice-based research, the concept of emotional expression choreography was defined as the sequence and timing of the upper and lower face regions (see Figure 1). Through iterative studio practice, a range of emotional expressions were animated, with the choreography manipulated by the lead author (who acted as a practitioner-researcher). The practitioner-researcher evaluated the potential effect of choreography variation through reflective practice, and predicted how clear and authentic various choreography configurations would be when shown to audiences. Animations and predictions were assessed through a series of experiments, where participants rated animations under controlled conditions. Not only did these

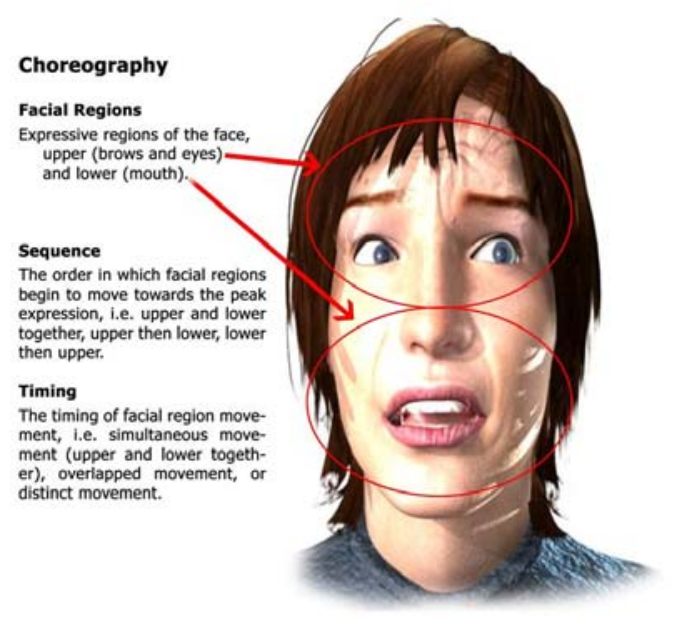

Figure 1: Emotional expression choreography

experiments demonstrate that variation in expression choreography could have a significant effect on perception, but in most cases the artistic predictions were shown to hold true (Sloan et al, 2010). Qualitative studies, using phenomenological methods of data recording and analysis, explored the interpretations of student animators after similar encounters with facial animation using emotional expression choreography. Consensus between participants was found in key areas. Additionally, focussing exercises involving audiences revealed how observers might interpret the deeper context of choreographed animation, which offered a more detailed picture of the effect of emotional expression choreography on perception.

At the conclusion of the Emotional Avatars research, many key areas were identified for future exploration. In particular, it was proposed that more in-depth practice-based studies should be carried out in order to explore the aesthetics of dynamic facial expressions, and to identify the most authentic choreographies of facial movement for a wider range of subtle expressions.

\section{References}

SloAn, R.J.S., Robinson, B., Scott-Brown, K. Moore, F. And CoOK, M. 2010. Emotional Facial Expression Choreography. Computer Animation and Virtual Worlds, 21, 3-4, 201-213.

Sullivan, G. 2009. Art Practice as Research. $2^{\text {nd }}$ ed. Thousand Oaks, CA: SAGE.

Thomas, F., AND Johnston, O. 1981. The Illusion of Life. New York, NY: Disney Editions. 